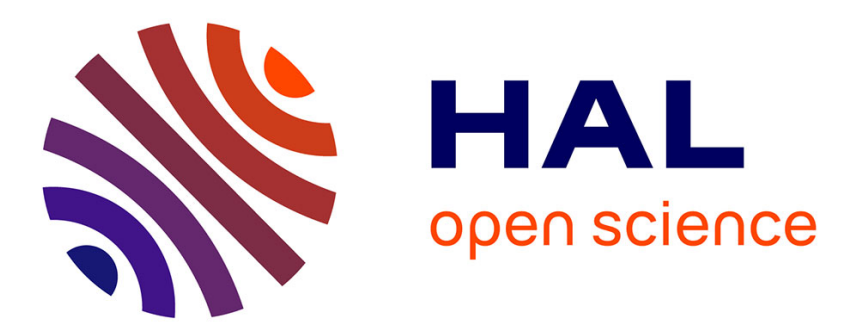

\title{
Adaptive control of Lipschitz time-delay systems by sigma modification with application to neuronal population dynamics
}

Jakub Orlowski, Antoine Chaillet, Alain Destexhe, Mario Sigalotti

\section{To cite this version:}

Jakub Orlowski, Antoine Chaillet, Alain Destexhe, Mario Sigalotti. Adaptive control of Lipschitz time-delay systems by sigma modification with application to neuronal population dynamics. Systems and Control Letters, 2022, 159, pp.105082. 10.1016/j.sysconle.2021.105082 . hal-03468630

\section{HAL Id: hal-03468630 \\ https://hal.inria.fr/hal-03468630}

Submitted on 3 Feb 2022

HAL is a multi-disciplinary open access archive for the deposit and dissemination of scientific research documents, whether they are published or not. The documents may come from teaching and research institutions in France or abroad, or from public or private research centers.
L'archive ouverte pluridisciplinaire HAL, est destinée au dépôt et à la diffusion de documents scientifiques de niveau recherche, publiés ou non, émanant des établissements d'enseignement et de recherche français ou étrangers, des laboratoires publics ou privés. 


\title{
Adaptive control of Lipschitz time-delay systems by sigma modification with application to neuronal population dynamics*
}

\author{
Jakub Orłowski ${ }^{\dagger}$ Antoine Chaillet, Alain Destexhe, Mario Sigalotti
}

February 3, 2022

\begin{abstract}
Adaptive control using the $\sigma$-modification provides an easily implementable way to stabilize systems with uncertain or fluctuating parameters. Motivated by a specific application from neuroscience, we extend here this methodology to nonlinear time-delay systems ruled by globally Lipschitz dynamics. In order to make the result more handy in practice, we provide an explicit construction of a LyapunovKrasovskii functional (LKF) with linear bounds and strict dissipation rate based on the knowledge of an LKF with quadratic bounds and point-wise dissipation rate. When applied to a model of neuronal populations involved in Parkinson's disease, the benefits with respect to a pure proportional stabilization scheme are discussed through numerical simulations.
\end{abstract}

\section{Introduction}

Stabilization by proportional feedback requires setting the feedback gain to an appropriate value, which sometimes can be challenging when faced

\footnotetext{
${ }^{*}$ This paper has received support from the Institute for Control and Decision of Paris Saclay (iCODE) and Digiteo.

${ }^{\dagger}$ Neuromuscular Systems Laboratory, School of Electrical \& Electronic Engineering, University College Dublin, Dublin, Ireland

${ }^{\ddagger}$ Université Paris-Saclay, CNRS, CentraleSupélec, Laboratoire des signaux et systèmes, 91190 Gif-sur-Yvette, France

${ }^{\S}$ Institut Universitaire de France

${ }^{\mathbb{I}}$ Université Paris-Saclay, CNRS, Institute of Neuroscience (NeuroPSI), Gif-sur-Yvette, France

" Sorbonne Université, Inria, CNRS, Laboratoire Jacques-Louis Lions (LJLL), Paris, France
} 
with unmodelled dynamics and unknown or time-varying system parameters. Adaptive control sidesteps those issues by designing controllers that automatically adjust their gains. For high-gain stabilizable systems, a naive approach is to initialize the control gain to a sufficiently low value and then to progressively increase it until the control objective is reached. This method, however, prevents the gain from decreasing and is therefore unable to correct for overestimation or to respond to changing parameters. Moreover, it can lead to unbounded growth of the control gain (parameter drift), even in the presence of vanishing disturbances [13].

One of the solutions to address this robustness issue is the $\sigma$-modification $[19,13]$. It consists in adding a leakage term to the update law of the control gain. This modification was shown to guarantee boundedness of solutions for certain classes of linear [12] and nonlinear [7] systems, as well as convergence of the error mean value to a residual set, whose size can be made arbitrarily small by an appropriate choice of the tuning parameter $\sigma[10,12]$.

While $\sigma$-modification has been applied to systems with input and measurement delays $[11,31,3]$, it was not yet shown to control nonlinear timedelay systems. In this work, we make a first step in this direction, by extending $\sigma$-modification to time-delay systems ruled by globally Lipschitz dynamics. Our main requirement is the existence of a Lyapunov-Krasovskii functional (LKF) with linear bounds and whose derivative has strict dissipation rate (dissipation involving the LKF itself) along the solutions of the system under high-gain proportional feedback. Since this requirement can be hard to check in practice, we also provide a constructive way to generate such an LKF based on an LKF with quadratic bounds whose derivative involves merely the current value of the solution (point-wise dissipation).

A particular field in which system parameters are highly uncertain and fluctuating is the one of biological systems. The original motivation for the present work is the study of a neuronal population model originally proposed in [20]. Under conditions explored in [20, 24, 26], this model exhibits oscillatory behavior, representing pathological $\beta$ brain oscillations (10-30 $\mathrm{Hz}$ ) observed in Parkinson's disease. In this system, time delays arise due to the non-instantaneous communication between neurons of the populations involved. Despite those delays, this model is known to be stabilizable by scalar high-gain proportional feedback, which gave rise to novel deep brain stimulation strategies for the treatment of parkinsonian symptoms $[8,25,2]$. By adapting the control gain in real time, the $\sigma$-modification strategy employed here is expected to offer better battery life, fewer side effects, and better adaptation to changing conditions than purely proportional control. While the present study focuses on analytical results on a neuronal 
population model, an extensive performance comparison between the two approaches was recently made on a detailed numerical model comprised of individual neurons [6].

It is worth stressing that a preliminary version of this work was presented in [22]. Nevertheless, the proof there relied on a result on partial stability published in [32] which turned out to be incorrect as we proved through a counter-example in [21]. Using a significantly different proof, the present paper corrects this flaw and additionally ensures some robustness to exogenous disturbances.

Notation. Given $x \in \mathbb{R}^{n},|x|$ denotes its Euclidean norm. $\mathcal{D}$ denotes the set of all signals $d: \mathbb{R}_{\geq 0} \rightarrow \mathbb{R}$ that are measurable and locally essentially bounded. Given a set $I \subset \mathbb{R}$ and a measurable essentially bounded signal

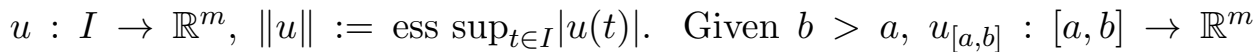
denotes the function defined as $u_{[a, b]}(t)=u(t)$ for all $t \in[a, b]$. Given $X \subset \mathbb{R}^{n}, C(I, X)$ denotes the set of all continuous functions $\phi: I \rightarrow X$ and, given $\bar{r}>0, \mathcal{C}:=C([-\bar{r}, 0], \mathbb{R})$ endowed with the sup norm. Given a signal $x:[-\bar{r},+\infty) \rightarrow \mathbb{R}^{n}$ and a time $t \geq 0, x_{t} \in \mathcal{C}^{n}$ denotes the history function: $x_{t}(s):=x(t+s)$ for all $s \in[-\bar{r}, 0]$.

\section{A motivating neuroscience application}

We start by introducing the problem that originally motivated the present work. Consider the following model of two interacting neuronal populations:

$$
\begin{aligned}
& \tau_{1} \dot{x}_{1}(t)=-x_{1}(t)+S_{1}\left(c_{11} x_{1}\left(t-r_{11}\right)-c_{12} x_{2}\left(t-r_{12}\right)+u(t)+d_{1}(t)+I_{1}\right), \\
& \tau_{2} \dot{x}_{2}(t)=-x_{2}(t)+S_{2}\left(c_{21} x_{1}\left(t-r_{21}\right)-c_{22} x_{2}\left(t-r_{22}\right)+d_{2}(t)+I_{2}\right),
\end{aligned}
$$

where $x_{1}(t)$ and $x_{2}(t)$ represent the instantaneous firing rate of populations 1 and 2 at time $t$, meaning the number of spikes emitted by their neurons per time unit. For each $i, j \in\{1,2\}, c_{i j} \geq 0$ represents the synaptic coupling strength from population $j$ to population $i$, whereas $r_{i j} \geq 0$ models axonal propagation delays from population $j$ to population $i$. Population 1 is excitatory whereas population 2 is inhibitory, as indicated through the sign with which they enter the dynamics. $\tau_{1}, \tau_{2}>0$ are time constants. $S_{1}, S_{2}: \mathbb{R} \rightarrow \mathbb{R}_{\geq 0}$ are the activation functions: they are typically globally Lipschitz (often picked as sigmoids or rectification functions in computational neuroscience literature). $u$ is a scalar control input modelling the 
impact of an artificial stimulator (for instance in deep brain stimulation). Finally, $I_{1}, I_{2} \geq 0$ represent the steady background influence of the major input structures to the modelled network, while $d_{1}, d_{2} \in \mathcal{D}$ represent exogenous disturbances that may result from variations around these steady values.

This model is based on the classical Wilson and Cowan population model [33]. It was used in [20] to explain the origin of pathological oscillations involved in Parkinson's disease (although no control input was considered in that reference) and further analysed in $[23,26]$. It was then employed in [8] to show that proportional control locally stabilizes the system when $d_{1}=d_{2} \equiv 0$, thus disrupting pathological oscillations. This result was later extended in [2] to global exponential stability.

In this paper, we will consider system (1) re-centered at the origin, which can be achieved by the change of state variables $x \leftrightarrow x-\bar{x}$, where $\bar{x}$ is any equilibrium of interest for (1) for $u, d_{1}, d_{2} \equiv 0$. In these new coordinates, the system becomes

$$
\begin{aligned}
& \tau_{1} \dot{x}_{1}(t)=-x_{1}(t)+S_{1}\left(c_{11} x_{1}\left(t-r_{11}\right)-c_{12} x_{2}\left(t-r_{12}\right)+u(t)+d_{1}(t)\right), \\
& \tau_{2} \dot{x}_{2}(t)=-x_{2}(t)+S_{2}\left(c_{21} x_{1}\left(t-r_{21}\right)-c_{22} x_{2}\left(t-r_{22}\right)+d_{2}(t)\right)
\end{aligned}
$$

where $S_{1}, S_{2}: \mathbb{R} \rightarrow \mathbb{R}$ are still globally Lipschitz but now satisfy $S_{1}(0)=$ $S_{2}(0)=0$, witnessing the fact that (2) now has an equilibrium at the orgin for $u, d_{1}, d_{2} \equiv 0$. As a result, $x_{1}$ and $x_{2}$ no longer represent the firing rates of the modelled structures but rather their values relative to the steady state. In particular, they may take negative values.

The population model (2) is a rough approximation of the microscopic dynamics resulting from neuronal activity. Estimation of its parameters based on electrophysiological recordings is often a hard task, and gives rise to significant uncertainty, particularly on the shape of the activation functions $S_{i}$ and on the synaptic weights $c_{i j}$. The transmission delays $r_{i j}$ can be known with a decent amount of accuracy, based on the mean signal propagation speed along the neuron's axon and the average distance between the considered structures. Likewise, the time constants $\tau_{i}$ should have values similar to the cell membrane time constant, which has been measured experimentally in many types of neurons. But even in those relatively straightforward cases, the estimated values would at best be crude approximations.

The result in [2, Proposition 1] ascertains that, no matter the exact 
values of the system's parameters, a proportional control of the form

$$
u(t)=-\theta x_{1}(t)
$$

always achieves exponential stability for $\theta$ sufficiently large (in the absence of disturbances), provided that $S_{2}$ has Lipschitz constant $\ell_{2}$ satisfying $c_{22} \ell_{2}<$ 1. Nevertheless, the estimation of such a proportional gain $\theta$ heavily depends on these uncertain parameters and and the value obtained in [2] is very conservative (in the sense that stability could be achieved with a much lower value than this theoretical guarantee).

Thus, the problem we want to address here is the following.

Problem statement. Despite the inherent uncertainty on the parameters of (2), automatically regulate the value of $\theta$ in (3) such that the closedloop system (2)-(3) has no steady-state oscillations when $d_{1}=d_{2} \equiv 0$ and preserves this property up to an error proportional to $\left\|d_{1}\right\|$ and $\left\|d_{2}\right\|$ in their presence.

The natural way to address this problem is to rely on adaptive control, which has been partially extended to time-delay systems, particularly for input delays [16], linear systems [35, 5, 1], or systems in triangular form $[17,15,34]$. Nevertheless, since uncertainty on the dynamics in (2) may affect any parameter involved and the very shape of the functions $S_{1}$ and $S_{2}$, the representation of (2) in strict feedback form becomes problematic. More crucially, due to its medical nature, this application requires a simple and robust strategy that limits the reliance on accurate parameter estimation as much as possible. These two constraints prevent the use of the cited works, and we rather opt for the so-called $\sigma$-modification originally introduced in [13]. Due to the presence of delays in the considered dynamics, we need to first develop a theory for such class of systems.

\section{Sigma modification for globally Lipschitz time- delay systems}

\subsection{Driver's derivative}

We start by recalling the notion of derivative introduced in [4]. Consider a nonlinear time-delay system

$$
\dot{x}(t)=f_{0}\left(x_{t}\right),
$$


where $x_{t} \in \mathcal{C}^{n}$ is the state of the system and $f_{0}: \mathcal{C}^{n} \rightarrow \mathbb{R}^{n}$ is Lipschitz continuous on bounded sets. Given any continuous function $V: \mathcal{C}^{n} \rightarrow \mathbb{R}$, its Driver derivative along the solutions of (4) is defined, for all $\phi \in \mathcal{C}^{n}$, as

$$
D_{(4)}^{+} V(\phi):=\limsup _{\tau \rightarrow 0^{+}} \frac{V\left(\phi_{\tau}^{\star}\right)-V(\phi)}{\tau}
$$

where, for all $\tau \in(0, \bar{r}), \phi_{\tau}^{\star} \in \mathcal{C}^{n}$ is defined as

$$
\phi_{\tau}^{\star}(s):= \begin{cases}\phi(s+\tau) & \text { if } s \in[-\bar{r},-\tau) \\ \phi(0)+f_{0}(\phi)(s+\tau) & \text { if } s \in[-\tau, 0] .\end{cases}
$$

As shown in [27], if $V$ is Lipschitz continuous on bounded sets and $t \mapsto x(t)$ is a solution of (4), then the Driver derivative of $V$ computed at $x_{t}$ coincides for almost every $t$ with the upper-right Dini derivative of $t \mapsto V\left(x_{t}\right)$.

\subsection{Stabilizability by proportional feedback}

Now, consider a control system

$$
\begin{aligned}
\dot{x}(t) & =f\left(x_{t}, u(t), d(t)\right) \\
y(t) & =h\left(x_{t}\right),
\end{aligned}
$$

where $u: \mathbb{R}_{\geq 0} \rightarrow \mathbb{R}$ denotes the control input, $d \in \mathcal{D}^{m}$ denotes a disturbance, $y$ is a scalar measured output, $h: \mathcal{C}^{n} \rightarrow \mathbb{R}$ is continuous and zero at zero, and the vector field $f: \mathcal{C}^{n} \times \mathbb{R} \times \mathbb{R}^{m} \rightarrow \mathbb{R}^{n}$ satisfies the following.

Assumption 1 (Global Lipschitz). $f$ is globally Lipschitz and satisfies $f(0,0,0)=0$.

In the absence of disturbances, this system is assumed to be globally exponentially stabilizable by proportional output feedback $u(t)=-\theta y(t)$, as stated next.

Assumption 2 (High-gain prop. stabilizability). There exist $\theta^{*} \geq 0$, a globally Lipschitz continuous functional $\mathcal{V}: \mathcal{C}^{n} \rightarrow \mathbb{R}_{\geq 0}$, and $\underline{\alpha}, \bar{\alpha}, \alpha>0$ such that, for all $\phi \in \mathcal{C}^{n}$,

$$
\underline{\alpha}|\phi(0)| \leq \mathcal{V}(\phi) \leq \bar{\alpha}\|\phi\|,
$$

and, given any gain $\theta \geq \theta^{*}$, the Driver derivative of $\mathcal{V}$ along the solutions of the closed-loop disturbance-free system

$$
\dot{x}(t)=f\left(x_{t},-\theta y(t), 0\right)
$$

satisfies

$$
D_{(9)}^{+} \mathcal{V}(\phi) \leq-\alpha \mathcal{V}(\phi)
$$


For a fixed $\theta \geq \theta^{*}$, the existence of such LKF is equivalent to global exponential stability of (9) [29, Theorem 2.5]. Assumption 2 thus imposes that the system is globally exponentially stabilizable by high-gain proportional feedback on $y$. It is, however, a more restrictive assumption, as it requires that the $\operatorname{LKF} \mathcal{V}$ is common to all $\theta \geq \theta^{*}$.

It is worth stressing that Assumption 2 can sometimes be established despite severe uncertainties on the system. For instance, we will show in Section 5 that the neuronal population model (2) does satisfy it, provided that $\ell_{2} c_{22}<1$ (where $\ell_{2}$ denotes the Lipschitz constant of $S_{2}$ ), regardless of the values of the time constants $\tau_{i}$, of the synaptic weights $c_{11}, c_{12}$ and $c_{21}$ and of the delays $r_{i j}$ and independently of the specific shape of the activation functions $S_{i}$.

Nevertheless, the requirement (10) is sometimes hard to achieve in practice, as the dissipation rate is required to involve the whole LKF itself. Also, it is often more convenient to use quadratic bounds on the LKF, rather than the linear bounds required in (8). In Section 4, we provide constructive tools to ensure these requirements for a spectific class of LKF satisfying a pointwise dissipation inequality. More precisely, for a class of quadratic LKF whose dissipation rate involves only the current value of the state norm, we construct an LKF satisfying (8) and (10). See [2] for a more thorough discussion about deducing exponential stability from a point-wise dissipation rate.

\section{3 $\sigma$-modification}

In view of the strong uncertainty on the model considered in the Problem Statement and of the conservative (if at all available) estimates of $\theta^{*}$, we rely on adaptive control with $\sigma$-modification, meaning

$$
\begin{aligned}
u(t) & =-\theta(t) y(t) \\
\tau_{\theta} \dot{\theta}(t) & =\kappa(y(t))-\sigma \theta(t),
\end{aligned}
$$

where $\kappa: \mathbb{R} \rightarrow \mathbb{R}_{\geq 0}$ denotes a locally Lipschitz function satisfying

$$
\kappa(y) \geq \underline{\kappa}|y|, \quad \forall y \in \mathbb{R}
$$

for some $\underline{\kappa}>0$. The function $\kappa$ is a design parameter whose specific choice can improve closed-loop performance. While it is not necessary for $\kappa$ to be 0 at 0 , in most applications the simplest choice would be $\kappa(y)=|y|$. In that

case, with the control law (11), the control gain $\theta(t)$ increases whenever $|y(t)|>\sigma \theta(t)$ and decreases whenever $|y(t)|<\sigma \theta(t)$, meaning when $y(t)$ 
is sufficiently close to the origin (if $\sigma$ is thought of as a small parameter). These balanced effects are designed in such a way that $\theta(t)$ adjusts its value around the lowest gain ensuring that solutions remain in a $\sigma$-vicinity of the origin, as will be made more precise below.

As stressed in [10], $\sigma$-modification does not guarantee, in general, that the state reaches an arbitrarily small neighborhood of the origin (for $\sigma$ small enough) and stays there indefinitely, even in delay-free context. Our objective is thus to establish the following weaker property.

Definition 1 (Practical stability in the mean). System (7) in closed loop with (11) is said to be robustly practically stable in the mean if there exist $q, \lambda>0$ such that, for any $\sigma>0$ small enough, any initial conditions $x_{0} \in \mathcal{C}^{n}$, any $\theta_{0} \geq 0$, and any bounded disturbance $d \in \mathcal{D}^{m}$, its solutions satisfy

$$
f_{t}^{t+T}|x(\tau)| \mathrm{d} \tau \leq q\left[\frac{\left\|x_{0}\right\| e^{-\lambda t}+\|d\|+1}{T}+\sigma+f_{t}^{t+T}|d(\tau)| \mathrm{d} \tau\right], \quad \forall t, T>0 .
$$

In the above definition, $f$ denotes the average of the signal over the considered time window. More precisely, for any $z \in \mathcal{D}$,

$$
f_{t}^{t+T} z(\tau) \mathrm{d} \tau:=\frac{1}{T} \int_{t}^{t+T} z(\tau) \mathrm{d} \tau, \quad \forall t, T \geq 0 .
$$

This notation is employed for compactness purposes and also to bear in mind that $f_{t}^{t+T} z(\tau) \mathrm{d} \tau$ does not necessarily vanish when $T \rightarrow+\infty$.

The property introduced in Definition 1 is named by analogy to the $\sigma$ small in the mean-square sense property, described in [12, Definition A.5.7]. By comparison, $\sigma$-small in the mean square sense is a property of a signal defined for a fixed $\sigma$, assuring similar behavior of the squared $L^{2}$-norm over a time window of length $T$.

Despite its apparent complexity, robust practical stability in the mean contains relevant applicative features. In the absence of exogenous disturbances (meaning for $d \equiv 0$ ), it guarantees that the steady-state mean value of the state norm over a sufficiently long window is essentially proportional to $\sigma$. Since $\sigma$ is a tuning parameter that can be picked arbitrarily small, this justifies the "practical stability" terminology. In other words, given any $\varepsilon>0$, there exist $\sigma^{*}, T^{*}>0$ such that, for all $\sigma \in\left(0, \sigma^{*}\right]$ and all $T \geq T^{*}$, any solution of the disturbance-free system satisfies

$$
\limsup _{t \rightarrow+\infty} f_{t}^{t+T}|x(\tau)| \mathrm{d} \tau \leq \varepsilon .
$$


It also ensures that, in the presence of a disturbance, this qualitative steadystate behavior is preserved up to a term proportional to the steady-state average of the disturbance norm.

\subsection{Main result}

The main contribution of this paper is to show that any Lipschitz time-delay system that can be exponentially stabilized by proportional output feedback is made robustly practically stable in the mean by adaptive control with $\sigma$ modification. More precisely, we have the following result, whose proof is provided in Section 6.1.

Theorem 1 (Stability in the mean by $\sigma$-modification). Under Assumptions 1 and 2, consider any locally Lipschitz function $\kappa: \mathbb{R} \rightarrow \mathbb{R}_{\geq 0}$, satisfying (12) for some $\underline{\kappa}>0$. Then:

(i) system (7) in closed loop with (11) is robustly practically stable in the mean;

(ii) there exists a constant $q>0$ such that, for each $x_{0} \in \mathcal{C}^{n}$, each $\theta_{0} \geq 0$, each $d \in \mathcal{D}^{m}$, and each $\sigma \in\left[0, \alpha \tau_{\theta}\right]$, the corresponding solution satisfies

$$
|x(t)| \leq q\left[\left(\left\|x_{0}\right\|+R^{*}\left(\theta_{0}\right)\right) e^{-\alpha t}+1+\|d\|\right], \quad \forall t \geq 0,
$$

where $R^{*}\left(\theta_{0}\right):=\min \left\{\theta_{0}-\theta^{*} ; 0\right\}\left(\theta_{0}-\theta^{*}\right)$ and $\alpha$ and $\theta^{*}$ are as in Assumption 2.

Property $(\mathrm{ii})$ corresponds to ultimate boundedness: all solutions converge exponentially to a neighborhood of the origin whose size is larger for larger disturbance magnitudes. It is a "hard" bound, in the sense that it provides an upper bound on the state norm at all times. Nevertheless, unlike property $(i)$, it guarantees no increased precision when picking a smaller value for the parameter $\sigma$.

Property (ii) also shows that the transient overshoot is bounded by a term that depends linearly on the initial state and on a term that involves the initial value of the adaptive gain $\theta_{0}$. Note that if $\theta$ is initialized to a value higher than $\theta^{*}$, the term $R^{*}\left(\theta_{0}\right)$ is zero.

\section{Construction of a strict LKF with linear bounds}

As already stressed, the main difficulty in applying Theorem 1 is checking Assumption 2. Indeed, it requires not only a globally Lipschitz LKF with linear bounds, but also a dissipation rate proportional to the LKF itself. 
Following the terminology from [2], the term $\alpha \mathcal{V}(\phi)$ in (10) is called a strict dissipation rate. On the other hand, a point-wise dissipation rate refers to the weaker requirement that $D_{(9)}^{+} V(\phi) \leq-\alpha|\phi(0)|$ for some $\alpha>0$. In view of (8), any strict dissipation rate is a point-wise dissipation rate, but the converse is not true. Point-wise dissipation turns out to be generally easier to check in practice than strict dissipation, as will be seen in Section 5.

Similarly, it is often convenient to use LKF with quadratic bounds rather than with linear bounds, as required by (8). In particular, the following family of LKF has proved useful for a wide class of time-delay systems (including the neuronal population model (2)):

$$
V(\phi)=\phi(0)^{T} P \phi(0)+\sum_{(i, j) \in J} \lambda_{i j} \int_{-r_{i j}}^{0} \phi_{j}(s)^{2} \mathrm{~d} s,
$$

where $J \subseteq\{1, \ldots, l\} \times\{1, \ldots, n\}$ with $l \in \mathbb{N}, r_{i j} \in(0, \bar{r}], P \in \mathbb{R}^{n \times n}$ with $P=P^{T}>0$, and $\lambda_{i j} \geq 0$. Such LKF are thus quadratic functionals involving a non-delayed term plus integral terms involving the delays $r_{i j}$ appearing in the system dynamics.

In this section, we provide constructive tools to obtain a globally Lipschitz LKF with strict dissipation rate and linear bounds, from a quadratic LKF of the form (14) with point-wise dissipation rate. To that aim, consider a general family of parametrized time-delay systems

$$
\dot{x}(t)=g\left(x_{t}, \theta\right)
$$

where $\theta \in \Theta \subset \mathbb{R}, x_{t} \in \mathcal{C}^{n}$, and $g: \mathcal{C}^{n} \times \mathbb{R} \rightarrow \mathbb{R}^{n}$ satisfies the conditions for existence and uniqueness of solutions (see for instance [9, Theorem 2.3, p. $42])$. It is worth stressing that none of the results presented in this section require global Lipschitz continuity of $g$.

\subsection{From point-wise to strict dissipation}

Several tricks exist to obtain a strict dissipation rate from a point-wise one. One of these tricks consists in adding an exponential function in the kernel of the integral terms of (14), see for instance $[28,14,18]$. The next lemma, proved in Section 6.2, shows that this ad hoc trick actually works for any quadratic LKF of the form (14). In other words, for that class of LKF, this result provides a systematic constructive way to obtain a strict LKF based on an LKF with a merely point-wise dissipation rate. 
Lemma 1 (From point-wise to strict). Assume that there exist $\alpha>0$ and a functional $V: \mathcal{C}^{n} \rightarrow \mathbb{R}_{\geq 0}$ of the form (14) such that, for all $\phi \in \mathcal{C}^{n}$ and all $\theta \in \Theta$,

$$
D_{(15)}^{+} V(\phi) \leq-\alpha|\phi(0)|^{2} .
$$

Then there exist $c, \alpha_{0}>0$ and $p>1$ such that the functional defined as

$$
W(\phi)=\phi(0)^{T} P \phi(0)+p \sum_{(i, j) \in J} \lambda_{i j} \int_{-r_{i j}}^{0} e^{c s} \phi_{j}(s)^{2} \mathrm{~d} s
$$

satisfies, for all $\phi \in \mathcal{C}^{n}$ and all $\theta \in \Theta$,

$$
D_{(15)}^{+} W(\phi) \leq-\alpha_{0} W(\phi)
$$

\subsection{From quadratic bounds to linear bounds}

Now, in order to satisfy Assumption 2, we want to show that we can construct a globally Lipschitz LKF with linear bounds from an LKF with quadratic bounds. This is done with the following result.

Lemma 2 (From quadratic to linear bounds). Assume that there exist $\underline{\alpha}_{0}$, $\bar{\alpha}_{0}, \alpha_{0}>0$ and a functional $W: \mathcal{C}^{n} \rightarrow \mathbb{R}_{\geq 0}$ of the form (17) satisfying, for all $\phi \in \mathcal{C}^{n}$ and all $\theta \in \Theta$,

$$
\begin{gathered}
W(\phi)=0 \quad \Rightarrow \quad g(\phi, \theta)=0 \\
D_{(15)}^{+} W(\phi) \leq-\alpha_{0} W(\phi) .
\end{gathered}
$$

Then there exist $\underline{\alpha}, \bar{\alpha}, \alpha>0$ such that the functional $\mathcal{V}:=\sqrt{W}$ is globally Lipschitz and satisfies, for all $\phi \in \mathcal{C}^{n}$ and all $\theta \in \Theta$,

$$
\begin{aligned}
\underline{\alpha}|\phi(0)| & \leq \mathcal{V}(\phi) \leq \bar{\alpha}\|\phi\|, \\
D_{(15)}^{+} \mathcal{V}(\phi) & \leq-\alpha \mathcal{V}(\phi) .
\end{aligned}
$$

The proof of this lemma is provided in Section 6.3. Although this result is not surprising, the main difficulty in that proof is to show that the constructed LKF $\mathcal{V}=\sqrt{W}$ is globally Lipschitz.

\section{$4.3 \sigma$-modification with quadratic LKF and point-wise dissi- pation}

The above two lemmas allow to replace Assumption 2 by the following. 
Assumption 3 (High-gain proportional stabilizability). There exist $\theta^{*} \geq 0$, $\alpha>0$, and a functional $V: \mathcal{C}^{n} \rightarrow \mathbb{R}_{\geq 0}$ of the form (14) such that, given any gain $\theta \geq \theta^{*}$, the Driver derivative of $V$ along the solutions of the disturbancefree closed-loop system (9) satisfies

$$
D_{(9)}^{+} V(\phi) \leq-\alpha|\phi(0)|^{2} .
$$

Then we have the following corollary, which is more conservative than Theorem 1 (as it imposes a particular structure on the LKF) but easier to use in practice. It readily follows from Theorem 1 and Lemmas 1 and 2 .

Corollary 1 ( $\sigma$-modification, revisited). Under Assumptions 1 and 3, the conclusions $(i)$ and $($ ii) of Theorem 1 remain valid.

\section{Application to neuronal populations}

\subsection{Theoretical result}

We now apply these theoretical results to the model of neuronal populations introduced in Section 2. We consider the following adaptive control law:

$$
\begin{aligned}
u(t) & =-\theta(t) x_{1}(t) \\
\tau_{\theta} \dot{\theta}(t) & =\left|x_{1}(t)\right|-\sigma \theta(t),
\end{aligned}
$$

with $\tau_{\theta}>0$ and $\sigma \geq 0$, which is nothing else than (11) with $\kappa\left(x_{1}\right)=\left|x_{1}\right|$. This adaptive control law fits the constraint imposed in Problem Statement, as it relies only on the measurement of $x_{1}$. It is expected to self-tune the value of the gain $\theta$ to achieve a correct oscillation attenuation with reasonable over-estimation due to the presence of the dissipation term $-\sigma \theta(t)$ in (22b), thus leading to lower energy consumption than proportional control, and to cope better with parameter modifications corresponding for instance to disease evolution, as will be discussed through simulations in Section 5.2. Based on the development presented in the previous sections, we have the following result whose proof is provided in Section 6.4.

Proposition 1 (Neuronal populations). For each $i, j \in\{1,2\}$, let $c_{i j}, r_{i j} \geq$ 0 and $\tau_{i}>0$. Assume that $S_{i}: \mathbb{R} \rightarrow \mathbb{R}$ are non-decreasing globally Lipschitz functions satisfying $S_{i}(0)=0$ and that the Lipschitz constant $\ell_{2}$ of $S_{2}$ satisfies

$$
c_{22} \ell_{2}<1 \text {. }
$$


Then system (2) in closed loop with (22) is globally practically stable in the mean and there exists $\bar{\sigma}>0$ such that, for any $\sigma \in[0, \bar{\sigma})$, its solutions are bounded.

In the absence of disturbances (namely, for $d_{1}=d_{2} \equiv 0$ ), this result ensures that the mean amplitude steady-state oscillations over a sufficiently long time window can be made arbitrarily small by choosing $\sigma$ small enough. In the presence of disturbances, the same qualitative behavior is preserved up to a term proportional to their magnitude. While the adaptive law does not guarantee exact disappearance of steady-state oscillations (unlike proportional control), we believe that this result satisfactorily answers the problem stated in Section 2.

It is worth stressing that Proposition 1 requires very little knowledge on the dynamics ruling (2). No information on the delays $r_{i j}$ or on the time constants $\tau_{i}$ is required. None of the synaptic weights $c_{i j}$ needs to be known in the design of the control law. In the same way, the activation function $S_{1}$ can be any non-decreasing Lipschitz function satisfying $S_{1}(0)=$ 0 . The only constraint lies in condition (23), which allows a greater $c_{22}$ if the Lipschitz constant of $S_{2}$ is smaller. Note that this condition requires no precise knowledge on the shape of $S_{2}$, but only on its maximal slope.

Condition (23) was also the main requirement in [2, Proposition 1] to ensure global exponential stability of (22) using the proportional control $u=$ $-\theta x_{1}$. With no extra assumption, Proposition 1 states that this oscillation disruption can also be achieved using an adaptive law.

\subsection{Simulations}

In order to illustrate the behavior of (2) in closed loop with the adaptive controller (22) and compare its performance with respect to proportional control, we run numerical simulations with the following activation functions $S_{i}$ :

$$
S_{i}(x):=\frac{m_{i} b_{i}}{b_{i}+e^{\frac{-4 x}{m_{i}}}\left(m_{i}-b_{i}\right)}-b_{i} .
$$

For any $m_{i}, b_{i}>0$, this function is nondecreasing, Lipschitz with Lipschitz constant 1 and satisfies $S_{i}(0)=0$, as required by Proposition 1 .

The parameters used in the simulations are: $r_{11}=0 \mathrm{~ms}, r_{12}=r_{21}=$ $6 \mathrm{~ms}, r_{22}=4 \mathrm{~ms}, c_{11}=0, c_{12}=10.7, c_{21}=20, c_{22}=0.9, m_{1}=300$, $m_{2}=400, b_{1}=17, b_{2}=75, \tau_{1}=6 \mathrm{~ms}, \tau_{2}=14 \mathrm{~ms}$, and $\tau_{\theta}=20 \mathrm{~ms}$. In particular, condition (23) is fulfilled. All simulations were performed in Python using forward Euler method with time-step $0.05 \mathrm{~ms}$. 
The neuronal population (2) was simulated under the $\sigma$-modification adaptive control law (22). The disturbance $d$ in (2) was taken as a piecewise constant function, randomly sampled every $2 \mathrm{~ms}$ from a uniform distribution on $[-D, D]^{2}, D \geq 0$. In all simulations, the adaptive gain was initialized with $\theta_{0}=0$.

The performance of $(22)$ in the absence of disturbance $(D=0)$ is presented in Figure 1 for a controller parameter $\sigma=0.03$. Initially $(t<200 \mathrm{~ms})$, the controller is turned off and the system exhibits self-sustained oscillations, characteristic of parkinsonian activity [20]. At $t=200 \mathrm{~ms}$, the controller is switched on. The adaptive gain $\theta$ progressively increases and oscillations in both $x_{1}$ and $x_{2}$ are significantly reduced. The gain eventually settles at a steady-state value, slightly below the minimal gain that assures global exponential stability, explaining the persistence of the remaining steady-state oscillations. The convergence of $\theta$ close to the minimal value guaranteeing oscillations disruptions suggests that the proposed adaptive controller self-tunes the gain in a way that limits the intensity of the delivered stimulation, which constitutes a promising feature in terms of side effects and energy consumption.

In order to systematically study the behavior of the closed-loop system, Figure 2 reports the magnitude of the state oscillations, calculated as the peak-to-peak amplitude of $|x(t)|$ over the final $2000 \mathrm{~ms}$ of the simulation averaged over 20 runs, as a function of the control parameter $\sigma$ and for different disturbance amplitudes $D$. It is clearly visible that the amplitude of steady-state oscillations can be arbitrarily reduced (up to a factor "proportional" to the disturbance amplitude) by an appropriate choice of $\sigma$. This steady-state amplitude reaches 0 for $\sigma=0$ and $D=0$. This confirms the theoretical expectations of Proposition 1.

The adaptive controller is also capable of dealing with changes in the system itself, which may result from progression of the disease or changes in the electrode-tissue interface. In order to illustrate this, we have simulated the progression of the disease and the corresponding increase of the pathological oscillations by increasing the synaptic coupling between the two populations, in the same spirit as in the original paper [20]. Figure 3 shows the system (2) in closed loop with $(22)$ in the presence of disturbances $(D=75)$. In the initial part of the simulation $(t<1.25 \mathrm{~s})$, both the adaptive controller (22) (top plot) and a purely proportional controller with $\theta=55$ (middle plot) show similar performance and the adaptive gain $\theta$ actually converges to 55. However, after the coupling constants $c_{12}$ and $c_{21}$ are increased at $t=1.25 \mathrm{~s}$, the adaptive controller outperforms the proportional controller in terms of oscillations attenuation by automatically adjusting its gain $\theta$ to 

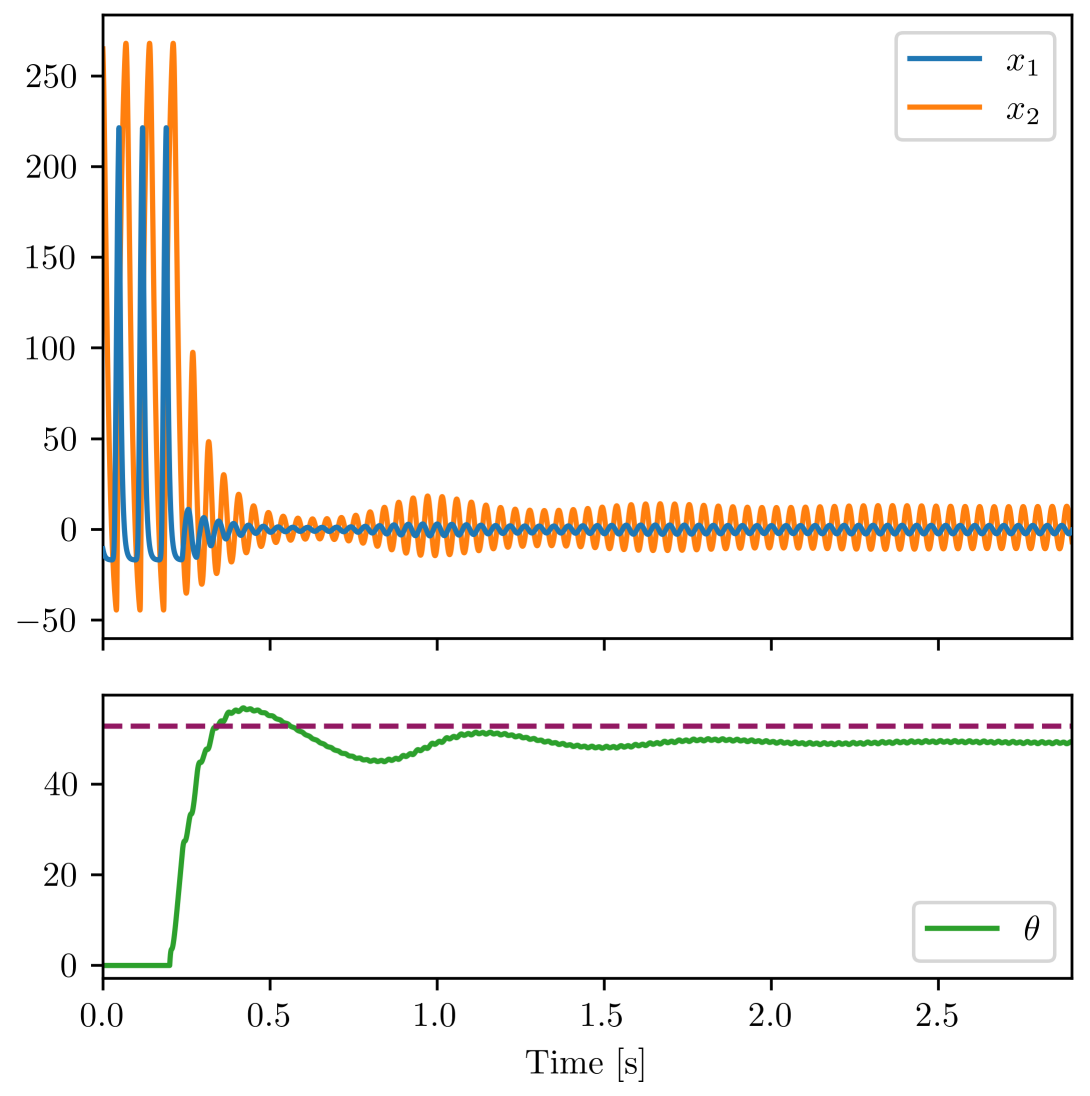

Figure 1: Illustration of the adaptive controller (22) applied to (2) without disturbances. For $t<200 \mathrm{~ms}$ the controller is off and the system exhibits self-sustained oscillations. At $t=200 \mathrm{~ms}$ the controller is turned on and the oscillations amplitude is greatly reduced. The dashed purple line in the bottom plot is the approximate value of the minimal constant stabilizing gain $\theta$ for the system with no disturbance (estimated via simulations). It should not be confused with the theoretical estimate $\theta^{*}$ obtained from (50), which is approximately $1.4710^{8}$ (not shown in the plot). 


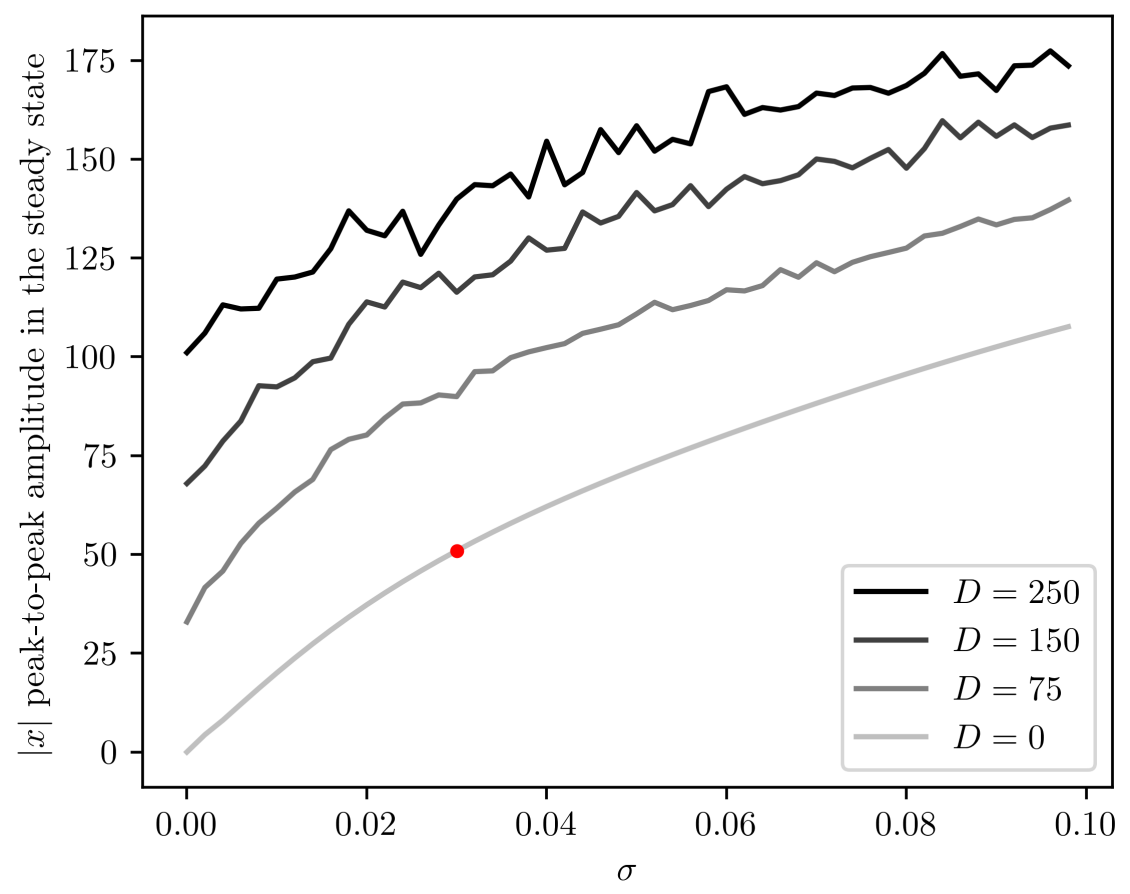

Figure 2: Steady-state peak-to-peak amplitude of the state norm $|x(t)|$ as a function of the control parameter $\sigma$, for different amplitudes $D$ of the additive disturbance $d$. The red dot on the plot represents the conditions corresponding to Figure 1. 
a higher value. In fact, the $L_{2}$ norm of the state variable $x$ over the final $1000 \mathrm{~ms}$ of the simulation under adaptive stimulation is approximately half the value achieved under proportional stimulation (16.8 versus 47.6 in the case presented in Figure 3).

A much deeper discussion of the benefits of the $\sigma$-modification adaptive control for closed-loop deep brain stimulation in Parkinson's disease, in terms of energy consumption, robustness to changing conditions, and practical implementability, is available in [6]. In that paper, the proposed strategy was tested on a detailed numerical model of the brain structures involved (networks of conductance-based neurons, rather than population dynamics).

\subsection{Unknown equilibrium}

Assumption 1 stipulates that the vector field $f$ governing the behaviour of the considered system has an equilibrium at 0 . In Section 2 we claim that the system can be re-centered at the origin by a change of variables, so that $x$ represent variations of the firing rates around that equilibrium. Nevertheless, the proposed adaptive control law (22) is based on $x_{1}$, and not on the actual firing rate in the STN population. In other words, the implementation of the adaptive control law implicitly assumes knowledge of the equilibrium value of the system, which might be hard, if not impossible, to find in the context of the neural populations that are the motivating example for this paper.

This practical limitation, while not yet overcome by our theoretical results, can be circumvented by either estimating the equilibrium using a lowpass filtered version of $x_{1}$, as in [30] or using an altogether different error signal $y$ in (11).

One such $y(t)$ that shows promising result in simulations is the "instantaneous" peak-to-peak amplitude of the STN firing rate, namely

$$
y(t)=\max _{s \in[-T, 0]} x_{1}(t+s)-\min _{s \in[-T, 0]} x_{1}(t+s),
$$

where $T$ is a window length to be chosen and $x_{1}$ is here understood as the first state variable of the (not re-centered) model (1). Ideally, the value of $T$ should be the length of one cycle of the pathological oscillation that we want to disrupt, to preserve the responsiveness of the controller while making sure that we respond to the actual magnitude of the oscillations. Note that such a peak-to-peak amplitude of the STN firing rate is realistically accessible to measurement.

The behaviour of the original system (1) in closed loop (11) with $\kappa(y)=$ $|y|$, using this peak-to-peak signal amplitude is illustrated in Figure 4. The 

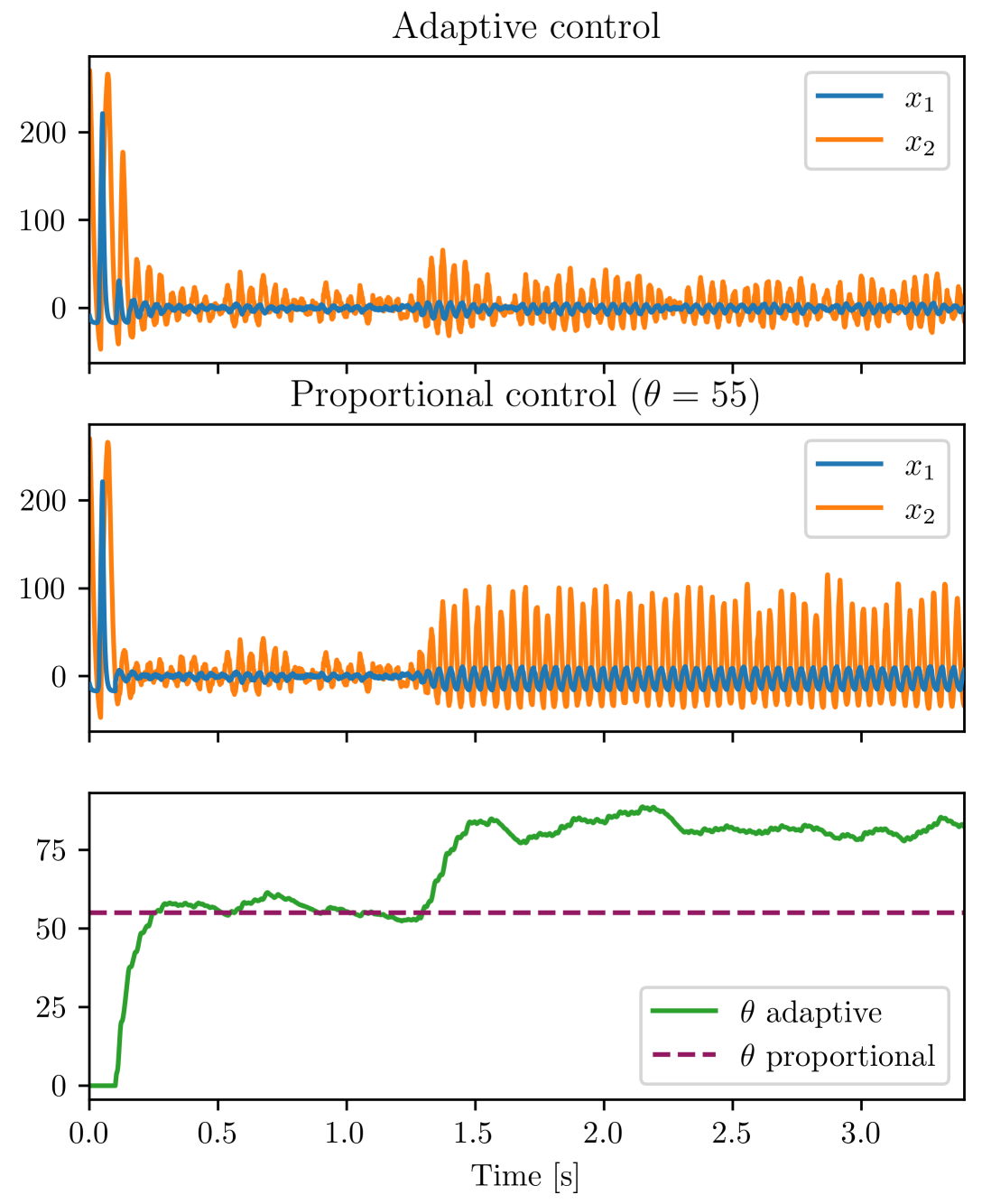

Figure 3: Comparison of the response to change in system parameters under adaptive and proportional control. The initial conditions are the same as for simulations used to obtain Figure 1 but with additional noise $(D=75)$. At $t=1.25 \mathrm{~s}$, the connectivity constants $c_{12}$ and $c_{21}$ are increased by $50 \%$ and $10 \%$, respectively. The adaptive controller (top plot) disrupts the oscillations to a higher degree than the purely proportional controller (middle plot) by increasing the value of the controller gain $\theta$ (bottom plot). 
values represented in the plot correspond to the firing rates $x_{1}$ and $x_{2}$ in spikes per second, with the activation functions

$$
S_{i}(x):=\frac{m_{i} b_{i}}{b_{i}+e^{\frac{-4 x}{m_{i}}}\left(m_{i}-b_{i}\right)}
$$

and the exogenous constant inputs set to $I_{1}=65.34$ and $I_{2}=-30.2$, which corresponds to the diseased dynamics described in [20]. The window length for calculating the peak-to-peak amplitude is $T=80 \mathrm{~ms}$, to capture a full pseudo-period of the $\simeq 13 \mathrm{~Hz}$ steady-state oscillation and the remaining parameters are identical to the parameters used for Figure 1. We can see that the controller with the modified error signal achieves the goal of reducing the magnitude of the pathological oscillations and autonomously regulates the proportional gain $\theta$ to a level that assures disruption of the oscillations.

\section{Proofs}

\subsection{Proof of Theorem 1}

System (7) in closed loop with (11) can be compactly written as

$$
\begin{aligned}
\dot{x}(t) & =f\left(x_{t},-\theta y(t), d(t)\right) \\
\dot{\theta}(t) & =\kappa(y(t))-\sigma \theta(t) .
\end{aligned}
$$

Denoting by $\theta_{t}$ the history function for $\theta$, we can see the above closed-loop system as evolving in $\mathcal{C}^{n+1}$ and having $\left(x_{t}, \theta_{t}\right)$ as state. Consider the LKF $\mathcal{W}: \mathcal{C}^{n+1} \rightarrow \mathbb{R}_{\geq 0}$ defined as

$$
\mathcal{W}(\phi, \vartheta)=\mathcal{V}(\phi)+\rho \frac{\tau_{\theta}}{2} R^{*}(\vartheta(0))
$$

where $\mathcal{V}$ is as in Assumption 2 and $\rho>0$ is a parameter to be chosen later. In the whole proof, $\phi$ should be thought of as $x_{t}$ when evaluated along the solutions, whereas $\vartheta$ represents $\theta_{t}$, and $\delta$ stands for $d(t)$. We recall that $R^{*}(\theta)=\min \left\{\theta-\theta^{*} ; 0\right\}\left(\theta-\theta^{*}\right) \geq 0$ for all $\theta \in \mathbb{R}$. Then, in view of $(8), \mathcal{W}$ satisfies

$$
\underline{\alpha}|\phi(0)| \leq \mathcal{W}(\phi, \vartheta) \leq \bar{\alpha}\|\phi\|+\frac{\rho \tau_{\theta}}{2} R^{*}(\vartheta(0)) .
$$

Whenever $\vartheta(0) \geq \theta^{*}$, it holds that $\mathcal{W}(\phi, \vartheta)=\mathcal{V}(\phi)$ and, since $\left.\frac{d R^{*}(\theta)}{d \theta}\right|_{\theta=\theta^{*}}=$ 0 , it also holds that $D_{(26)}^{+} \mathcal{W}=D_{(26 a)}^{+} \mathcal{V}$. Moreover, Assumption 2 guarantees 

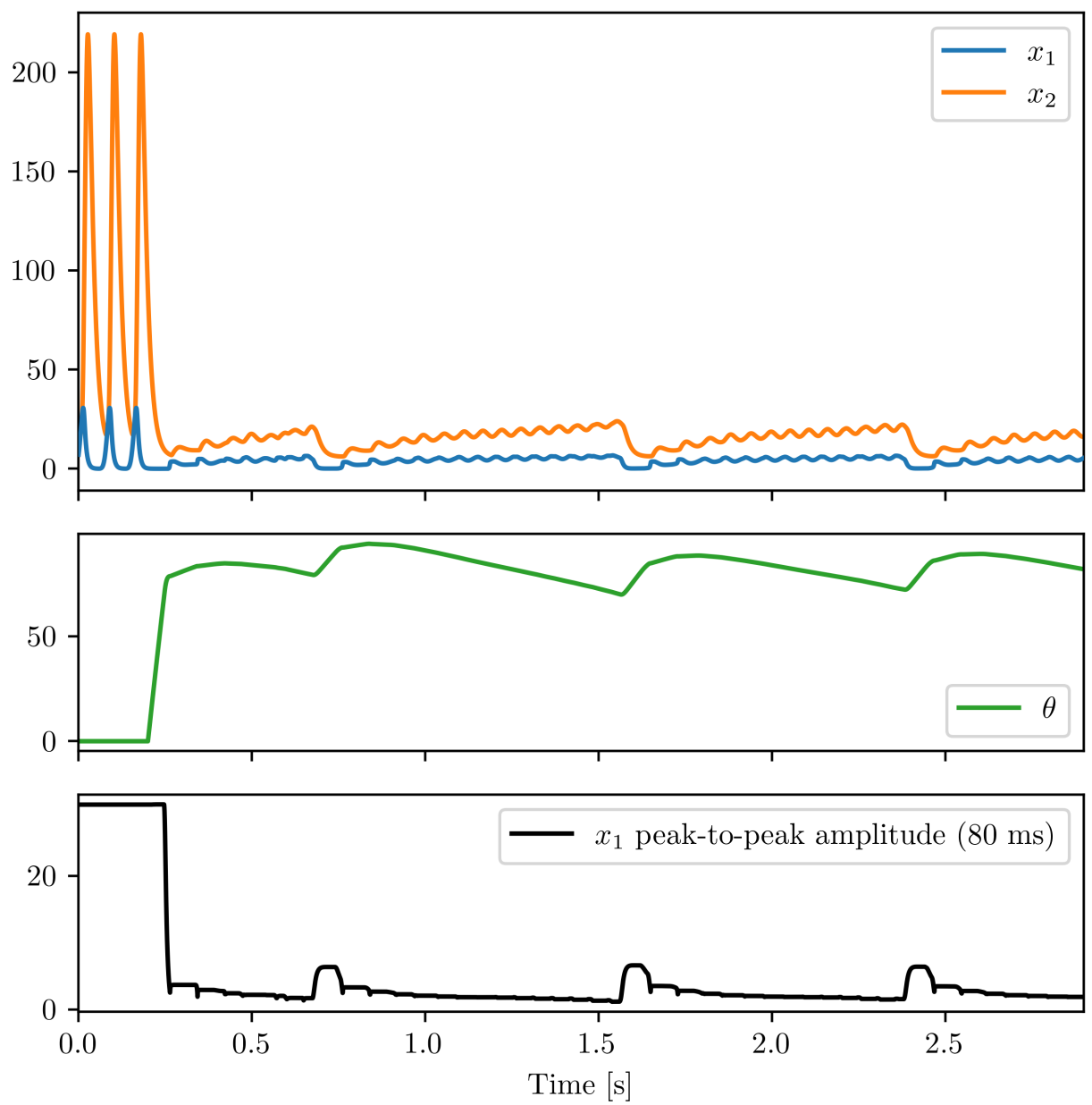

Figure 4: Illustration of the adaptive controller (11) applied to (1) with the original activation functions from [20] and the system output $y(t)$ calculated as in (24) with $T=80 \mathrm{~ms}$. The top plot shows the firing rates $x_{1}$ and $x_{2}$, the middle plot the time evolution of the proportional gain $\theta$ and the bottom plot the output $y$, which is used in the controller (11) in lieu of $x_{1}(t)$. 
that, along the disturbance-free system,

$$
D_{(9)}^{+} \mathcal{V} \leq-\alpha \mathcal{V}(\phi)
$$

Recalling the definition of Driver's derivative (5)-(6), define

$$
\phi_{\tau, \vartheta, \delta}^{\star}(s):= \begin{cases}\phi(s+\tau) & \text { if } s \in[-\bar{r},-\tau) \\ \phi(0)+f(\phi,-\vartheta(0) h(\phi), \delta)(s+\tau) & \text { if } s \in[-\tau, 0],\end{cases}
$$

where we explicitly mentioned the $\vartheta$ - and $\delta$-dependency in the subscript. Observing that the vector field of (26a) coincides with that of (9) whenever $d=0$, it holds that, for $\vartheta(0) \geq \theta^{*}$,

$$
\begin{aligned}
& D_{(26)}^{+} \mathcal{W}=D_{(26 \mathrm{a})}^{+} \mathcal{V} \\
& \quad=\limsup _{\tau \rightarrow 0^{+}} \frac{1}{\tau}\left(\mathcal{V}\left(\phi_{\tau, \vartheta, 0}^{\star}\right)-\mathcal{V}(\phi)+\mathcal{V}\left(\phi_{\tau, \vartheta, \delta}^{\star}\right)-\mathcal{V}\left(\phi_{\tau, \vartheta, 0}^{\star}\right)\right) \\
& \quad \leq D_{(9)}^{+} \mathcal{V}(\phi)+\limsup _{\tau \rightarrow 0^{+}} \frac{1}{\tau}\left|\mathcal{V}\left(\phi_{\tau, \vartheta, \delta}^{\star}\right)-\mathcal{V}\left(\phi_{\tau, \vartheta, 0}^{\star}\right)\right| \\
& \quad \leq-\alpha \mathcal{V}(\phi)+\limsup _{\tau \rightarrow 0^{+}} \frac{\ell \mathcal{V}}{\tau}\left\|\phi_{\tau, \vartheta, \delta}^{\star}-\phi_{\tau, \vartheta, 0}^{\star}\right\|,
\end{aligned}
$$

where the last inequality comes from $(29), \ell_{\mathcal{V}}>0$ denoting the Lipschitz constant of $\mathcal{V}$ as ensured by Assumption 2. Since $\phi_{\tau, \vartheta, \delta}^{\star}$ and $\phi_{\tau, \vartheta, 0}^{\star}$ coincide over $[-r,-\tau)$, it holds that

$$
\begin{aligned}
\left\|\phi_{\tau, \vartheta, \delta}^{\star}-\phi_{\tau, \vartheta, 0}^{\star}\right\| & =\sup _{s \in[-\tau, 0]}\left|\phi_{\tau, \vartheta, \delta}^{\star}(s)-\phi_{\tau, \vartheta, 0}^{\star}(s)\right| \\
& =\sup _{s \in[-\tau, 0]}|f(\phi,-\vartheta(0) h(\phi), \delta)-f(\phi,-\vartheta(0) h(\phi), 0)|(s+\tau) .
\end{aligned}
$$

Since $f$ is globally Lipschitz (Assumption 1), it follows that there exists a constant $\ell_{f}>0$ such that

$$
\left\|\phi_{\tau, \vartheta, \delta}^{\star}-\phi_{\tau, \vartheta, 0}^{\star}\right\| \leq \sup _{s \in[-\tau, 0]} \ell_{f}|\delta|(s+\tau) \leq \ell_{f}|\delta| \tau .
$$

Combining this with (30), we conclude that

$$
\vartheta(0) \geq \theta^{*} \quad \Rightarrow \quad D_{(26)}^{+} \mathcal{W} \leq-\alpha \mathcal{V}(\phi)+\ell_{\mathcal{V}} \ell_{f}|\delta| .
$$

On the other hand, when $\vartheta(0)<\theta^{*}, \mathcal{W}(\phi, \vartheta)=\mathcal{V}(\phi)+\frac{\rho \tau_{\theta}}{2}\left(\vartheta(0)-\theta^{*}\right)^{2}$ and its derivative along solutions of (26) satisfies

$$
\begin{aligned}
& D_{(26)}^{+} \mathcal{W}=D_{(26 \mathrm{a})}^{+} \mathcal{V}+\rho\left(\vartheta(0)-\theta^{*}\right)(\kappa(h(\phi))-\sigma \vartheta(0)) \\
& =D_{(26 \mathrm{a})}^{+} \mathcal{V}+\rho\left(-\left|\vartheta(0)-\theta^{*}\right| \kappa(h(\phi))-\sigma \vartheta(0)^{2}+\sigma \vartheta(0) \theta^{*}\right) \\
& \leq D_{(26 \mathrm{a})}^{+} \mathcal{V}+\rho\left(-\underline{\kappa}\left|\vartheta(0)-\theta^{*}\right||h(\phi)|-\frac{\sigma}{2} \vartheta(0)^{2}+\frac{\sigma}{2} \theta^{* 2}\right)
\end{aligned}
$$


where we used (12). With a slight abuse of notation, let $\phi_{\tau, \theta^{*}, \delta}^{\star}$ denote $\phi_{\tau, \vartheta, \delta}^{\star}$ for $\vartheta(\cdot) \equiv \theta^{*}$. Proceeding as above, it holds that

$$
\begin{aligned}
& D_{(26 \mathrm{a})}^{+} \mathcal{V} \\
& \quad=\limsup _{\tau \rightarrow 0^{+}} \frac{1}{\tau}\left(\mathcal{V}\left(\phi_{\tau, \theta^{*}, 0}^{\star}\right)-\mathcal{V}(\phi)+\mathcal{V}\left(\phi_{\tau, \vartheta, \delta}^{\star}\right)-\mathcal{V}\left(\phi_{\tau, \theta^{*}, 0}^{\star}\right)\right) \\
& \quad \leq-\alpha \mathcal{V}(\phi)+\limsup _{\tau \rightarrow 0^{+}} \frac{1}{\tau}\left|\mathcal{V}\left(\phi_{\tau, \vartheta, \delta}^{\star}\right)-\mathcal{V}\left(\phi_{\tau, \theta^{*}, 0}^{\star}\right)\right| \\
& \quad \leq-\alpha \mathcal{V}(\phi)+\limsup _{\tau \rightarrow 0^{+}} \frac{\ell \mathcal{V}}{\tau}\left\|\phi_{\tau, \vartheta, \delta}^{\star}-\phi_{\tau, \theta^{*}, 0}^{\star}\right\|,
\end{aligned}
$$

where the negative term comes from Assumption 2. Since $\phi_{\tau, \vartheta, \delta}^{\star}$ and $\phi_{\tau, \theta^{*}, 0}^{\star}$ coincide over $[-r,-\tau)$, it holds that

$$
\begin{aligned}
\left\|\phi_{\tau, \vartheta, \delta}^{\star}-\phi_{\tau, \theta^{*}, 0}^{\star}\right\| & =\sup _{s \in[-\tau, 0]}\left|\phi_{\tau, \vartheta, \delta}^{\star}(s)-\phi_{\tau, \theta^{*}, 0}^{\star}(s)\right| \\
& =\sup _{s \in[-\tau, 0]}\left|f(\phi,-\vartheta(0) h(\phi), \delta)-f\left(\phi,-\theta^{*} h(\phi), 0\right)\right|(s+\tau) \\
& \leq \ell_{f}\left(\left|\vartheta(0)-\theta^{*}\right||h(\phi)|+|\delta|\right) \tau .
\end{aligned}
$$

Plugging that back into (33), we get

$$
D_{(26 \mathrm{a})}^{+} \mathcal{V} \leq-\alpha \mathcal{V}(\phi)+\ell_{\mathcal{V}} \ell_{f}\left(\left|\vartheta(0)-\theta^{*}\right||h(\phi)|+|\delta|\right) .
$$

It follows from (32) that

$$
\begin{aligned}
D_{(26)}^{+} \mathcal{W} \leq & -\alpha \mathcal{V}(\phi)+\ell_{\mathcal{V}} \ell_{f}\left(\left|\vartheta(0)-\theta^{*}\right||h(\phi)|+|\delta|\right) \\
& +\rho\left(-\underline{\kappa}\left|\vartheta(0)-\theta^{*}\right||h(\phi)|-\frac{\sigma}{2} \vartheta(0)^{2}+\frac{\sigma}{2} \theta^{* 2}\right) .
\end{aligned}
$$

By picking $\rho=\ell_{\mathcal{V}} \ell_{f} / \underline{\kappa}$, we obtain that

$$
D_{(26)}^{+} \mathcal{W} \leq-\alpha \mathcal{V}(\phi)+\frac{\rho}{2} \sigma \theta^{* 2}+\ell_{\mathcal{V}} \ell_{f}|\delta|
$$

whenever $\vartheta(0)<\theta^{*}$. In view of $(31)$, this bound is also valid for the case when $\vartheta(0) \geq \theta^{*}$. In other words, (34) is true everywhere.

Now we proceed to show $(i i)$. The set

$$
E:=\left\{(\phi, \vartheta) \in \mathcal{C}^{n+1}: \vartheta(0) \geq 0\right\}
$$

is forward invariant for (26). To see this, observe that $\theta\left(t_{0}\right) \geq 0$ for some $t_{0} \geq 0$ implies by (26b) and the non-negativity of $\kappa$ that $\theta(t) \geq 0$ for all 
$t \geq t_{0}$. In addition, if $\vartheta(0) \geq \theta^{*}$ then $R^{*}(\vartheta(0))=0$ whereas, for $\vartheta(0)<\theta^{*}$, $R^{*}(\vartheta(0))=\left(\vartheta(0)-\theta^{*}\right)^{2} \leq \theta^{* 2}$ for all $(\phi, \vartheta) \in E$. It follows from (28) that, for all $(\phi, \vartheta) \in E$,

$$
\mathcal{V}(\phi) \leq \mathcal{W}(\phi, \vartheta) \leq \mathcal{V}(\phi)+\frac{\rho \tau_{\theta}}{2} \theta^{* 2}
$$

Hence, (34) ensures that, for all $(\phi, \vartheta) \in E$,

$$
D_{(26)}^{+} \mathcal{W} \leq-\alpha \mathcal{W}(\phi)+\alpha \rho \tau_{\theta} \theta^{* 2}+\ell_{\mathcal{V}} \ell_{f}|\delta|,
$$

where we used the inequality $\sigma \leq \alpha \tau_{\theta}$ as assumed in the statement. Integrating along solutions of $(26)$ over their maximal interval of existence $[0, b)$, $b \in(0,+\infty]$, we get that

$$
\mathcal{W}\left(x_{t}, \theta_{t}\right) \leq \mathcal{W}\left(x_{0}, \theta_{0}\right) e^{-\alpha t}+\rho \tau_{\theta} \theta^{* 2}+\frac{\ell_{\mathcal{V}} \ell_{f}}{\alpha}\|d\| .
$$

Using (28), we conclude that, for all $t \in[0, b)$,

$$
\underline{\alpha}|x(t)| \leq\left(\bar{\alpha}\left\|x_{0}\right\|+\frac{\rho \tau_{\theta}}{2} R^{*}(\vartheta(0))\right) e^{-\alpha t}+\rho \theta^{* 2} \tau_{\theta}+\frac{\ell_{\mathcal{V}} \ell_{f}}{\alpha}\|d\| .
$$

This shows in particular that no finite escape time can occur, meaning that $b=+\infty$ (see $[9$, Theorem 3.1, p. 42]). Item (ii) follows by picking any $q$ large enough that

$$
q \geq \frac{1}{\underline{\alpha}} \max \left\{\bar{\alpha}, \frac{\rho \tau_{\theta}}{2}, \rho \theta^{* 2} \tau_{\theta}, \frac{\ell \mathcal{\nu} \ell_{f}}{\alpha}\right\}
$$

Next we proceed to show $(i)$. From (34) and (28), it holds that

$$
D_{(26)}^{+} \mathcal{W} \leq-\alpha \underline{\alpha}|\phi(0)|+\frac{\rho}{2} \sigma \theta^{* 2}+\ell_{\mathcal{V}} \ell_{f}|\delta|,
$$

meaning that, along solutions of (26),

$$
D_{(26)}^{+} \mathcal{W}\left(x_{t}, \theta_{t}\right) \leq-\alpha \underline{\alpha}|x(t)|+\frac{\rho}{2} \sigma \theta^{* 2}+\ell_{\mathcal{V}} \ell_{f}|d(t)| .
$$

Integrating both sides we get that, for any $t, T \geq 0$,

$$
\alpha \underline{\alpha} \int_{t}^{t+T}|x(\tau)| \mathrm{d} \tau \leq \mathcal{W}\left(x_{t}, \theta_{t}\right)+\frac{\rho}{2} \sigma \theta^{* 2} T+\ell_{\mathcal{V}} \ell_{f} \int_{t}^{t+T}|d(\tau)| \mathrm{d} \tau .
$$


Using (36) and then (35), it follows that

$$
\begin{aligned}
\alpha \underline{\alpha} \int_{t}^{t+T}|x(\tau)| \mathrm{d} \tau \leq & \mathcal{W}\left(x_{0}, \theta_{0}\right) e^{-\alpha t}+\rho \theta^{* 2} \tau_{\theta}+\frac{\ell_{\mathcal{V}} \ell_{f}}{\alpha}\|d\| \\
& +\frac{\rho}{2} \sigma \theta^{* 2} T+\ell_{\mathcal{V}} \ell_{f} \int_{t}^{t+T}|d(\tau)| \mathrm{d} \tau \\
\leq & \left(\bar{\alpha}\left\|x_{0}\right\|+\frac{\rho \tau_{\theta}}{2} \theta^{* 2}\right) e^{-\alpha t}+\rho \theta^{* 2} \tau_{\theta}+\frac{\ell_{\mathcal{V}} \ell_{f}}{\alpha}\|d\| \\
& +\frac{\rho}{2} \sigma \theta^{* 2} T+\ell_{\mathcal{V}} \ell_{f} \int_{t}^{t+T}|d(\tau)| \mathrm{d} \tau .
\end{aligned}
$$

Rearranging terms we obtain

$$
\begin{aligned}
f_{t}^{t+T}|x(\tau)| \mathrm{d} \tau \leq & \frac{1}{\alpha \underline{\alpha} T}\left[\bar{\alpha}\left\|x_{0}\right\| e^{-\alpha t}+2 \rho \tau_{\theta} \theta^{* 2}+\frac{\ell_{\mathcal{\nu} \ell_{f}}}{\alpha}\|d\|\right] \\
& +\frac{\rho \theta^{* 2}}{2 \alpha \underline{\alpha}} \sigma+\frac{\ell_{\mathcal{\nu} \ell_{f}}}{\alpha \underline{\alpha}} f_{t}^{t+T}|d(\tau)| \mathrm{d} \tau .
\end{aligned}
$$

This establishes $(i)$ for any $q$ satisfying

$$
q \geq \frac{1}{\alpha \underline{\alpha}} \max \left\{\bar{\alpha}, 2 \rho \tau_{\theta} \theta^{* 2}, \frac{\ell_{\mathcal{V}} \ell_{f}}{\alpha}, \frac{\rho \theta^{* 2}}{2}, \ell_{\mathcal{V}} \ell_{f}\right\} .
$$

The conclusion follows by picking $q$ large enough to fulfill both this and (37).

\subsection{Proof of Lemma 1}

In view of (14) and (17), $W$ reads

$$
W(\phi)=V(\phi)+\sum_{(i, j) \in J} \lambda_{i j} \int_{-r_{i j}}^{0}\left(p e^{c s}-1\right) \phi_{j}(s)^{2} \mathrm{~d} s .
$$

By definition of Driver's derivative and by the sub-additivity of the lim sup, we have that

$$
D_{(15)}^{+} W(\phi) \leq D_{(15)}^{+} V(\phi)+\sum_{(i, j) \in J} \lambda_{i j} D_{(15)}^{+} W_{i j}(\phi),
$$

where $W_{i j}(\phi):=\int_{-r_{i j}}^{0}\left(p e^{c s}-1\right) \phi_{j}(s)^{2} \mathrm{~d} s$. Given $\phi \in \mathcal{C}^{n}$ and $\theta \in \Theta$, define $\hat{\phi}:[-r,+\infty) \rightarrow \mathbb{R}^{n}$ by $\hat{\phi}(s)=\phi(s)$ for $s \leq 0$ and $\hat{\phi}(s)=\phi(0)+s g(\phi, \theta)$ for $s>0$. From (6), it follows that $\hat{\phi}(\tau+s)=\phi_{\tau}^{\star}(s)$ for every $s \in[-\bar{r}, 0]$ and every $\tau \in[0, \bar{r}]$. 


\section{Consequently,}

$$
\begin{aligned}
& D_{(15)}^{+} W_{i j}(\phi) \\
& \quad=\limsup _{\tau \rightarrow 0^{+}} \frac{1}{\tau}\left(\int_{-r_{i j}}^{0}\left(p e^{c s}-1\right) \phi_{\tau, j}^{\star}(s)^{2} \mathrm{~d} s-\int_{-r_{i j}}^{0}\left(p e^{c s}-1\right) \phi_{j}(s)^{2} \mathrm{~d} s\right) \\
& \quad=\limsup _{\tau \rightarrow 0^{+}} \frac{1}{\tau}\left(\int_{-r_{i j}}^{0}\left(p e^{c s}-1\right) \hat{\phi}_{j}(s+\tau)^{2} \mathrm{~d} s-\int_{-r_{i j}}^{0}\left(p e^{c s}-1\right) \hat{\phi}_{j}(s)^{2} \mathrm{~d} s\right) \\
& \quad=\limsup _{\tau \rightarrow 0^{+}} \frac{1}{\tau}\left(\int_{\tau-r_{i j}}^{\tau}\left(p e^{c(s-\tau)}-1\right) \hat{\phi}_{j}(s)^{2} \mathrm{~d} s-\int_{-r_{i j}}^{0}\left(p e^{c s}-1\right) \hat{\phi}_{j}(s)^{2} \mathrm{~d} s\right) .
\end{aligned}
$$

Since $\hat{\phi}$ is continuous, the limsup on the right-hand side of (39) coincides with the upper-right derivative of $\tau \mapsto \int_{\tau-r_{i j}}^{\tau}\left(p e^{c(s-\tau)}-1\right) \hat{\phi}_{j}(s)^{2} \mathrm{~d} s$ at $\tau=$ 0 , that is,

$$
\begin{aligned}
D_{(15)}^{+} W_{i j}(\phi)= & (p-1) \phi_{j}(0)^{2}-\left(p e^{-c r_{i j}}-1\right) \phi_{j}\left(-r_{i j}\right)^{2} \\
& -c p \int_{-r_{i j}}^{0} e^{c s} \phi_{j}(s)^{2} \mathrm{~d} s .
\end{aligned}
$$

Combining (16), (38), and (40), we get that

$$
\begin{aligned}
D_{(15)}^{+} W(\phi) \leq & -\alpha|\phi(0)|^{2}+(p-1) \sum_{(i, j) \in J} \lambda_{i j} \phi_{j}(0)^{2} \\
& -\sum_{(i, j) \in J}\left(\alpha+\lambda_{i j}\left(p e^{-c r_{i j}}-1\right)\right) \phi_{j}\left(-r_{i j}\right)^{2} \\
& -c p \sum_{(i, j) \in J} \lambda_{i j} \int_{-r_{i j}}^{0} e^{c s} \phi_{j}(s)^{2} \mathrm{~d} s .
\end{aligned}
$$

Notice that, since $p>1$ by assumption,

$$
(p-1) \sum_{(i, j) \in J} \lambda_{i j} \phi_{j}(0)^{2} \leq(p-1) \bar{\lambda}|\phi(0)|^{2},
$$

where $\bar{\lambda}:=\max _{(i, j) \in J} \lambda_{i j}$. Pick $p>1$ in such a way that $\alpha-\bar{\lambda}(p-1) \geq \alpha / 2$ (a possible choice being $p=1+\alpha / 2 \bar{\lambda})$. Then, letting $p_{M}>0$ denote the maximal eigenvalue of $P$, we get that

$$
-\alpha|\phi(0)|^{2}+(p-1) \sum_{(i, j) \in J} \lambda_{i j} \phi_{j}(0)^{2} \leq-\frac{\alpha}{2 p_{M}} \phi(0)^{T} P \phi(0) .
$$


Pick $c>0$ in such a way that $\alpha+\lambda_{i j}\left(p e^{-c r_{i j}}-1\right) \geq 0$ for every $(i, j) \in J$ (for instance, we can choose $c=\ln (p) / \bar{r}$, where $\left.\bar{r}:=\max _{(i, j) \in J} r_{i j}>0\right)$. Then, substituting into (41), we get

$$
\begin{aligned}
D_{(15)}^{+} W & \leq-\frac{\alpha}{2 p_{M}} \phi(0)^{T} P \phi(0)-c p \sum_{(i, j) \in J} \int_{-r_{i j}}^{0} e^{c s} \phi_{j}(s)^{2} d s \\
& \leq-\min \left\{\frac{\alpha}{2 p_{M}}, c\right\} W(\phi) .
\end{aligned}
$$

The conclusion follows with

$$
\alpha_{0}:=\min \left\{\frac{\alpha}{2 p_{M}}, c\right\}=\min \left\{\frac{\alpha}{2 p_{M}}, \frac{1}{\bar{r}} \ln \left(1+\frac{\alpha}{2 \bar{\lambda}}\right)\right\} .
$$

\subsection{Proof of Lemma 2}

Les $W$ be as in (17). Then it readily holds that

$$
\underline{\alpha}_{0}|\phi(0)|^{2} \leq W(\phi) \leq \bar{\alpha}_{0}\|\phi\|^{2}
$$

with $\underline{\alpha}_{0}=p_{m}$ and $\bar{\alpha}_{0}=p_{M}+p \bar{\lambda} \bar{r} J^{\#}$, where $p_{m}$ and $p_{M}$ are respectively the minimum and maximum eigenvalue of $P, \bar{\lambda}=\max _{(i, j) \in J} \lambda_{i j}$, and $J^{\#}$ is the number of elements in $J$. Taking square root of both sides of the inequalities of (42) we recover (20a) with $\underline{\alpha}=\sqrt{\underline{\alpha}_{0}}$ and $\bar{\alpha}=\sqrt{\bar{\alpha}_{0}}$.

For all $\phi \in \mathcal{C}^{n}$ such that $W(\phi)=0,(18)$ ensures that $g(\phi, \theta)=0$ for every $\theta \in \Theta$. Computation of Driver's derivative leads to $D_{(15)}^{+} \mathcal{V}(\phi)=0$, which establishes (20b) given any $\alpha>0$ since $\mathcal{V}(\phi)=0$. For all $\phi$ such that $W(\phi)>0$, we rely on the following chain rule result.

Lemma 3. For every continuous nondecreasing $\varphi: \mathbb{R}_{\geq 0} \rightarrow \mathbb{R}$ and every locally Lipschitz function $W: \mathcal{C}^{n} \rightarrow \mathbb{R}$, the Driver derivative of $\varphi \circ W$ satisfies

$$
D_{(15)}^{+}(\varphi \circ W)(\phi) \leq D_{(15)}^{+} W(\phi) \varphi^{\prime}(W(\phi)),
$$

for all $\phi \in \mathcal{C}^{n}$ for which $\varphi$ is differentiable at $W(\phi)$.

The result is just an adaptation of [14, Lemma 6], which required in addition that $\varphi$ is continuously differentiable (in which case (43) holds for all $\left.\phi \in \mathcal{C}^{n}\right)$.

Recall that we are left to consider the case $W(\phi)>0$, for which $\varphi(\cdot)=\sqrt{ }$. is differentiable at $W(\phi)$. From Lemma 3 we get that

$$
D_{(15)}^{+} \mathcal{V}(\phi)=D_{(15)}^{+} \sqrt{W(\phi)} \leq \frac{1}{2 \sqrt{W(\phi)}} D_{(15)}^{+} W(\phi) .
$$


Using (15), it follows that

$$
D_{(15)}^{+} \mathcal{V}(\phi) \leq-\frac{\alpha_{0}}{2 \sqrt{W(\phi)}} W(\phi)=-\frac{\alpha_{0}}{2} \mathcal{V}(\phi),
$$

from which we deduce (20b) with $\alpha=\alpha_{0} / 2$.

We are only left to show that $\mathcal{V}$ is globally Lipschitz. To that aim, observe the following.

Fact 1. Given any $\phi_{0}, \phi_{1} \in \mathcal{C}^{n}$, the function $a:[0,1] \rightarrow \mathbb{R}_{\geq 0}$ defined as

$$
a(\xi):=\mathcal{V}\left(\phi_{0}+\xi\left(\phi_{1}-\phi_{0}\right)\right)
$$

is Lipschitz continuous on $[0,1]$.

Proof. For conciseness, let

$$
\phi_{\xi}:=\phi_{0}+\xi\left(\phi_{1}-\phi_{0}\right) .
$$

If $\phi_{\xi} \neq 0$ for every $\xi \in[0,1]$, then $a$ is Lipschitz as a composition of two Lipschitz functions ( $W$ restricted to a bounded set and square root on a closed interval not containing 0$)$. Otherwise, there exists $\bar{\xi} \in[0,1]$ such that $a(\bar{\xi})=0$. By considering the function $a(\cdot)$ separately on the two intervals $[0, \bar{\xi}]$ and $[\bar{\xi}, 1]$ we easily reduce the problem to the case where $\bar{\xi}=0$, i.e., $\phi_{0}=0$. Then, using (17), for any $\xi \in[0,1]$,

$$
a(\xi)=\sqrt{W\left(\xi \phi_{1}\right)}=\xi \sqrt{W\left(\phi_{1}\right)},
$$

proving the Lipschitz continuity of $a$.

From the definition of $a$ it holds that $\mathcal{V}\left(\phi_{1}\right)-\mathcal{V}\left(\phi_{0}\right)=a(1)-a(0)$. From Lemma $1, a^{\prime}$ is defined almost everywhere and $\mathcal{V}\left(\phi_{1}\right)-\mathcal{V}\left(\phi_{0}\right)=\int_{0}^{1} a^{\prime}(\xi) \mathrm{d} \xi$. In particular,

$$
\left|\mathcal{V}\left(\phi_{1}\right)-\mathcal{V}\left(\phi_{0}\right)\right| \leq \int_{0}^{1}\left|a^{\prime}(\xi)\right| \mathrm{d} \xi
$$

Let $\phi_{\xi}$ be defined as in (44). Wherever $\phi_{\xi} \neq 0$ the derivative of $a$ reads

$$
\begin{aligned}
a^{\prime}(\xi)= & \frac{\frac{\mathrm{d}}{\mathrm{d} \xi} W\left(\phi_{\xi}\right)}{2 \sqrt{W\left(\phi_{\xi}\right)}} \\
= & \frac{1}{\sqrt{W\left(\phi_{\xi}\right)}}\left(\phi_{\xi}^{T}(0) P\left(\phi_{1}(0)-\phi_{0}(0)\right)\right. \\
& \left.+p \sum_{(i, j) \in J} \lambda_{i j} \int_{-r_{i j}}^{0} e^{c s}\left(\phi_{1, j}-\phi_{0, j}\right)(s) \phi_{\xi, j}(s) \mathrm{d} s\right) .
\end{aligned}
$$


Letting $p_{m}$ and $p_{M}$ denote the minimal and maximal eigenvalues of $P$, it holds that

$$
\begin{aligned}
\left|\frac{\phi_{\xi}^{T}(0) P\left(\phi_{1}(0)-\phi_{0}(0)\right)}{\sqrt{W\left(\phi_{\xi}\right)}}\right| & \leq \frac{\left|\phi_{\xi}^{T}(0) P\left(\phi_{1}(0)-\phi_{0}(0)\right)\right|}{\sqrt{\phi_{\xi}^{T}(0) P \phi_{\xi}(0)}} \\
& \leq \frac{p_{M}\left|\phi_{\xi}(0)\right|\left|\phi_{1}(0)-\phi_{0}(0)\right|}{\sqrt{p_{m}}\left|\phi_{\xi}(0)\right|} \\
& \leq c_{0}\left\|\phi_{1}-\phi_{0}\right\|,
\end{aligned}
$$

where $c_{0}:=\frac{p_{M}}{\sqrt{p_{m}}}$. Similarly, for the integral terms:

$$
\begin{aligned}
I_{i j} & :=\left|\frac{\int_{-r_{i j}}^{0} e^{c s}\left(\phi_{1, j}-\phi_{0, j}\right)(s) \phi_{\xi, j}(s) d s}{\sqrt{W\left(\phi_{\xi}\right)}}\right| \\
& \leq \frac{1}{\sqrt{p \lambda_{i j}}} \frac{\left|\int_{-r_{i j}}^{0} e^{c s}\left(\phi_{1, j}-\phi_{0, j}\right)(s) \phi_{\xi, j}(s) d s\right|}{\sqrt{\int_{-r_{i j}}^{0} e^{c s} \phi_{\xi, j}(s)^{2} d s}} .
\end{aligned}
$$

By the Cauchy-Schwarz inequality, we have

$$
\begin{aligned}
& \left|\int_{-r_{i j}}^{0} e^{c s}\left(\phi_{1, j}-\phi_{0, j}\right)(s) \phi_{\xi, j}(s) \mathrm{d} s\right| \\
& \leq \sqrt{\int_{-r_{i j}}^{0} e^{c s} \phi_{\xi, j}(s)^{2} \mathrm{~d} s} \sqrt{\int_{-r_{i j}}^{0} e^{c s}\left(\phi_{1, j}-\phi_{0, j}\right)(s)^{2} \mathrm{~d} s},
\end{aligned}
$$

implying that

$$
\begin{aligned}
I_{i j} & \leq \frac{1}{\sqrt{p \lambda_{i j}}} \sqrt{\int_{-r_{i j}}^{0} e^{c s}\left(\phi_{1, j}-\phi_{0, j}\right)(s)^{2} \mathrm{~d} s} \\
& \leq \frac{r_{i j}}{\sqrt{p \lambda_{i j}}}\left\|\phi_{1, j}-\phi_{0, j}\right\| \\
& \leq \frac{n r_{i j}}{\sqrt{p \lambda_{i j}}}\left\|\phi_{1}-\phi_{0}\right\| .
\end{aligned}
$$

Combining (47), (48) and (49), we get from (46) that, for almost every $\xi \in[0,1]$,

$$
\left|a^{\prime}(\xi)\right| \leq\left(c_{0}+n \bar{r} \sqrt{p \bar{\lambda}}\right)\left\|\phi_{1}-\phi_{0}\right\|
$$

where $\bar{r}=\max _{(i, j) \in J} r_{i j}$ and $\bar{\lambda}=\max _{(i, j) \in J} \lambda_{i j}$. Global Lipschitz continuity of $\mathcal{V}$ then follows from (45). 


\subsection{Proof of Proposition 1}

It was shown in the proof of [2, Theorem 1] that there exists an LKF $V$, of the form (14), such that (21) holds with $\alpha=\frac{1-c_{22}}{2}$ for any $\theta \geq \theta^{*}$ with

$$
\theta^{*}:=8\left(c_{11}^{2}+\frac{4 c_{12}^{2} c_{21}^{2}}{\left(1-c_{22} \ell_{2}\right)^{2}}\right)
$$

This shows that Assumption 3 is satisfied. Assumption 1 is also fulfilled since $S_{1}$ and $S_{2}$ are globally Lipschitz. The conclusion follows from Corollary 1 .

\section{References}

[1] D. Bresch-Pietri, J. Chauvin, and N. Petit. Adaptive control scheme for uncertain time-delay systems. Automatica, 48(8):1536-1552, 2012.

[2] A. Chaillet, J. Orłowski, and P. Pepe. A relaxed Lyapunov-Krasovskii condition for global exponential stability of Lipschitz time-delay systems. In 58th IEEE Conference on Decision and Control, Dec. 2019.

[3] L. Chan, F. Naghdy, and D. Stirling. Application of adaptive controllers in teleoperation systems: A survey. IEEE Transactions on HumanMachine Systems, 44(3):337-352, 2014.

[4] R. D. Driver. Existence and stability of solutions of a delay-differential system. Arch. Rational Mech. Anal., 10(1):401-426, 1962.

[5] S. Evesque, A. Annaswamy, S. Niculescu, and A. Dowling. Adaptive control of a class of time-delay systems. J. Dyn. Sys., Meas., Control, 125(2):186-193, 2003.

[6] J. Fleming, J. Orłowski, M. M. Lowery, and A. Chaillet. Self-tuning deep brain stimulation controller for suppression of beta oscillations: analytical derivation and numerical validation. Frontiers in Neuroscience, 14:639, 2020.

[7] A. L. Fradkov, I. V. Miroshnik, and V. O. Nikiforov. Nonlinear and adaptive control of complex systems. Springer Science \& Business Media, 1999.

[8] I. Haidar, W. Pasillas-Lépine, A. Chaillet, E. Panteley, S. Palfi, and S. Senova. A firing-rate regulation strategy for closed-loop deep brain stimulation. Biological Cybernetics, 110(1):55-71, 2016. 
[9] J. Hale. Theory of functional differential equations. Springer-Verlag, New York-Heidelberg, 1977.

[10] L. Hsu and R. Costa. Bursting phenomena in continuous-time adaptive systems with a $\sigma$-modification. IEEE Transactions on Automatic Control, 32(1):84-86, jan 1987.

[11] J. Huang and F. L. Lewis. Neural-network predictive control for nonlinear dynamic systems with time-delay. IEEE Transactions on Neural Networks, 14(2):377-389, 2003.

[12] P. A. Ioannou and B. Fidan. Adaptive control tutorial. SIAM, 2006.

[13] P. A. Ioannou and P. V. Kokotovic. Instability analysis and improvement of robustness of adaptive control. Automatica, 20:583-594, 1984.

[14] H. Ito, P. Pepe, and Z. Jiang. A small-gain condition for iISS of interconnected retarded systems based on Lyapunov-Krasovskii functionals. Automatica, 46(10):1646-1656, Oct. 2010.

[15] X. Jiao and T. Shen. Adaptive feedback control of nonlinear time-delay systems: the lasalle-razumikhin-based approach. IEEE Trans. Autom. Control, 50(711):1909-1913, 2005.

[16] M. Krstić. Delay Compensation for Nonlinear, Adaptive, and PDE Systems. Brikhäuser, 2009.

[17] Z. Liu and Y. Wu. Universal strategies to explicit adaptive control of nonlinear time-delay systems with different structures. Automatica, 89:151-159, 2018.

[18] F. Mazenc, H. Ito, and P. Pepe. Construction of Lyapunov functionals for coupled differential and continuous time difference equations. In Proc. IEEE Conf. on Dec. and Control, pages 2245 - 2250, 2013.

[19] K. S. Narendra, S. S. Tripathi, G. Lueders, and P. Kudva. Adaptive control using Lyapunov's direct method. Technical report, Becton Center, Yale University, New Haven, Connecticut, 1971.

[20] A. J. Nevado-Holgado, J. R. Terry, and R. Bogacz. Conditions for the generation of beta oscillations in the subthalamic nucleus-globus pallidus network. Journal of Neuroscience, 30(37):12340-12352, 2010. 
[21] J. Orłowski, A. Chaillet, and M. Sigalotti. Counterexample to a Lyapunov condition for uniform asymptotic partial stability. IEEE Control Systems Letters, 4(2):397-401, 2020.

[22] J. Orłowski, A. Chaillet, M. Sigalotti, and A. Destexhe. Adaptive scheme for pathological oscillations disruption in a delayed neuronal population model. In Proc. IEEE Conf. on Decision and Control, Miami, USA, Dec. 2018.

[23] W. Pasillas-Lépine. Equilibrium and stability of Wilson and Cowan's time coarse graining model. In Proceedings of Mathematical Theory of Network Systems, 2012.

[24] W. Pasillas-Lépine. Delay-induced oscillations in Wilson and Cowan's model: An analysis of the subthalamo-pallidal feedback loop in healthy and parkinsonian subjects. Biological Cybernetics, 107(3):289-308, 2013.

[25] W. Pasillas-Lépine, I. Haidar, A. Chaillet, and E. Panteley. Closedloop deep brain stimulation based on firing-rate regulation. In Proc. 6th IEEE EMBS Conf. on Neural Engineering, San Diego, USA, 2013.

[26] A. Pavlides, S. John Hogan, and R. Bogacz. Improved conditions for the generation of beta oscillations in the subthalamic nucleus-globus pallidus network. European Journal of Neuroscience, 36(2):2229-2239, 2012.

[27] P. Pepe. On Liapunov-Krasovskii functionals under Carathéodory conditions. Automatica, 43(4):701-706, 2007.

[28] P. Pepe and Z. Jiang. A Lyapunov-Krasovskii methodology for ISS and iISS of time-delay systems. Systems \& Control Letters, 55(12):10061014, 2006.

[29] P. Pepe and I. Karafyllis. Converse Lyapunov-Krasovskii theorems for systems described by neutral functional differential equations in Hale's form. International Journal of Control, 86(2):232-243, Feb. 2013.

[30] K. Pyragas, V. Pyragas, I. Kiss, and J. Hudson. Adaptive control of unknown unstable steady states of dynamical systems. Physical Review E - Statistical Physics, Plasmas, Fluids, and Related Interdisciplinary Topics, 70(2):12, 2004. 
[31] D. Richert, C. J. B. Macnab, and J. K. Pieper. Adaptive control for haptics with time-delay. In 49th IEEE Conference on Decision and Control (CDC), pages 3650-3655. IEEE, 2010.

[32] V. I. Vorotnikov. Partial stability and control. Birkhauser, 1998.

[33] H. R. Wilson and J. D. Cowan. Excitatory and inhibitory interactions in localized populations of model neurons. Biophysical Journal, 12(1):124, 1972 .

[34] X. Zhang and Y. Lin. Adaptive output feedback control for a class of large-scale nonlinear time-delay systems. Automatica, 52:87-94, 2015.

[35] Y. Zhu and M. Krstić. Delay-Adaptive Linear Control. Princeton University Press, 2020. 